\title{
Jahrestagung der Gesellschaft für Interlinguistik e.V. Berlin, 10-12.11.2017
}

Vom 10. bis zum 12. November 2017 fand in Berlin-Schöneberg die 27. Jahrestagung der Gesellschaft für Interlinguistik e.V. (GIL) mit weit über vierzig Teilnehmern, überwiegend GIL-Mitgliedern, statt. Erstmals tagte die GIL in zentraler Lage in Berlin, was sich positiv auf die Teilnehmerzahl ausgewirkt haben dürfte.

Das Schwerpunktthema lautete „Mehrsprachigkeit in Europa“, wie üblich wurden aber nicht nur Vorträge über dieses Themenfeld, sondern auch zu vielen anderen Gebieten, die in einer breit verstandenen Interlinguistik behandelt werden, gehalten. Besonders gut ist es 2017 gelungen, Referenten und Publikum von außerhalb der GIL zu erreichen, sicher auch durch das nicht auf Plansprachen beschränkte Thema. Die folgenden Vorträge wurden gehalten (in alphabetischer Reihenfolge):

- Dr. Cyril Brosch (Leipzig): Zum Status der Mehrsprachigkeit im universitären Erasmus +-Programm - zur Erklärung des Widerspruchs zwischen der offiziellen Gleichberechtigung der Sprachen in Europa und der oft englischsprachigen Praxis unter Austauschstudenten.

- Prof. Dr. Liliana Ruth Feierstein (Berlin): Esperanto: Sprache und Utopie - zur Verortung des Esperantismus bzw. von Zamenhofs Weltbild im Kontext des jüdischen Diskurses über die Zukunft der europäischen Juden im 19. Jahrhundert.

- Prof. Dr. Sabine Fiedler (Leipzig): Esperanto und Mehrsprachigkeit-zur Haltung der Esperanto-Bewegung zur Mehrsprachigkeit im Wandel der Zeit und zu den mehrsprachigen Praktiken in der heutigen Esperanto-Sprachgemeinschaft.

- Dr. Michele Gazzola (Berlin): Sprachen und Arbeitsmarkt - zu aktuellen Studien zum Zusammenhang von Mehrsprachigkeit und besseren Chancen am Arbeitsmarkt (in Bezug auf Anstellung und Gehalt) und deren methodologischen Problemen.

- Dr. Bernd Krause (Forchheim): Verortung von Farben und und Bildung von Farbwörtern in ausgewählten Apriori-Planspracheprojekten - zur Verzahnung von Wortschatzbildung im genannten Bereich nach dem verfügbaren Weltwissen (Farbtheorien) im Wandel der Zeit.

- Krunoslav Puškar (Križevice, Kroatien): Lehnübersetzungen im Esperanto - zu Morphemkonstruktionen (v.a. Komposita) aus der Frühzeit des Esperanto, die überwiegend nach deutschen und russischen Vorbildern gebildet wurden, sowie deren Weiterentwicklung.

- Mira Sarikaya (Hamburg): Universalsprache bei Leibniz und der logische Aufbau der Welt - zu einem Dissertationsprojekt, in dem die von Leibniz skizzierte Idee der logischen Ordnung der Welt in einer Plansprache und deren Rezeption bei späteren Denkern wie Carnap untersucht werden soll.

- Dr. Britta Schneider (Berlin): Mehrsprachigkeit in Berlin, from above and from below'. Institutionen, Diskurse und Grassroots-Bewegungen - zu der widersprüchlichen Haltung zur Mehrsprachigkeit im öffentlichen Diskurs in Abhängigkeit vom 
Prestige der Sprachen und zu einem Fallbeispiel gelungener Förderung von Mehrsprachigkeit in Berlin-Pankow.

- Mag. Bernhard Tuider (Wien): Sammlung für Plansprachen und Esperanto-Museum - zu Geschichte, Zielen und Aufbau einerseits und andererseits aktuellen Entwicklungen und Projekten der Sammlung für Plansprachen, sowie zu den Plänen für die nächsten Jahre.

- Kristin Tytgat (Antwerpen): Mehrsprachigkeit und Sprachbewusstheit - zu den allgemeinen Problemen der Mehr- und Vielsprachigkeit, besonders in pädagogischer und sprachpsychologischer Hinsicht, unter Berücksichtigung der Erfahrungen mit Esperanto.

- Prof. Dr. Bengt-Arne Wickström (Budapest): Kostenbasierte Sprachplanung - zu den verschiedenen Arten ökonomischer Aufwendungen, die Mehrsprachigkeit bedeuten kann, und zur Berechnung, in welchen Fällen der Aufwand jeweils lohnenswert sein kann.

- Fritz Wollenberg (Berlin): Lebendige Esperanto-Kultur - präsentiert in dem neuen Jubiläumsbuch des EABB ,Esperanto - Sprache und Kultur in Berlin und Brandenburg - 111 Jahre " - zu einem kürzlich erschienenen umfangreichen Sammelband auch mit interlinguistisch interessanten Beiträgen.

- Louis von Wunsch-Rolshoven (Berlin): Das Bild des Esperanto in den Wissenschaften - zu auf Unkenntnis oder Missverständnissen beruhenden negativen Äußerungen zu Esperanto durch Wissenschaftler und den möglichen Ursachen dieser irrationalen Haltung.

Neben den wissenschaftlichen Vorträgen gab es am Samstagabend auch eine Demonstration des neuen Smartphone-Programms Amikumu zum Finden von Sprachpartnern (gerade auch in Plansprachen), das von seinem technischen Direktor Chuck Smith in lebhafter Diskussion vorgestellt wurde.

Die schriftlichen Ausarbeitungen der Vorträge sollen 2018 veröffentlicht werden. Ort dafür wird die neue Zeitschrift Jahrbuch der Gesellschaft für Interlinguistik (JGI) sein, deren erste Ausgabe im Oktober 2017 beim Leipziger Universitätsverlag erschienen ist und auf der Tagung vorgestellt wurde.

Weiterhin fanden die vereinsrechtliche Jahreshauptversammlung der GIL sowie eine Abschlussdiskussion zur Tagung statt, wo Themenvorschläge für die nächste Tagung im November 2018 gemacht wurden.

Cyril Robert Brosch „Mobilität und Inklusion in einem vielsprachigen Europa“ Universität Leipzig 